\title{
Erratum to: Rationale and design of oBservational clinical Research In chronic kidney disease patients with renal anemia: renal proGnosis in patients with Hyporesponsive anemia To Erythropoiesis-stimulating agents, darbepoetiN alfa (BRIGHTEN Trial)
}

\author{
Hideki Kato ${ }^{1} \cdot$ Masaomi Nangaku $^{1}$ - Hideki Hirakata ${ }^{2}$ 'Takashi Wada ${ }^{3} \cdot$ \\ Terumasa Hayashi $^{4}$ - Hiroshi Sato ${ }^{5}$ - Yasushi Yamazaki ${ }^{6}$ Takao Masaki ${ }^{7}$. \\ Tatsuo Kagimura ${ }^{8}$ - Hiroyasu Yamamoto ${ }^{9}$ Hiroki Hase ${ }^{10} \cdot$ Masahiro Kamouchi $^{11}$. \\ Enyu Imai $^{12}$ - Kyoichi Mizuno ${ }^{13}$ Manabu Iwasaki ${ }^{14}$ - Tadao Akizawa ${ }^{15}$. $^{13}$ \\ Yoshiharu Tsubakihara $^{16} \cdot$ Shoichi Maruyama $^{17} \cdot$ Ichiei Narita $^{18}$
}

Published online: 5 September 2017

(C) The Author(s) 2017

\section{Erratum to: Clin Exp Nephrol \\ DOI 10.1007/s10157-017-1427-4}

The article Rationale and design of oBservational clinical Research In chronic kidney disease patients with renal anemia: renal prognosis in patients with Hyporesponsive anemia To Erythropoiesis-stimulating agents, darbepoetiN alfa (BRIGHTEN Trial), written by Hideki Kato, Masaomi

The online version of the original article can be found under doi:10.1007/s10157-017-1427-4

Masaomi Nangaku

mnangaku-tky@umin.ac.jp

1 Division of Nephrology and Endocrinology,

The University of Tokyo Graduate School of Medicine,

7-3-1 Hongo, Bunkyo, Tokyo 113-8655, Japan

2 Fukuoka Renal Clinic, Fukuoka, Fukuoka, Japan

3 Department of Nephrology and Laboratory Medicine, Faculty of Medicine, Institute of Medical, Pharmaceutical and Health Sciences, Kanazawa University, Kanazawa, Ishikawa, Japan

4 Department of Kidney Disease and Hypertension, Osaka General Medical Centre, Sumiyoshi, Osaka, Japan

5 Division of Clinical Pharmacology and Therapeutics, Tohoku University Graduate School of Pharmaceutical Sciences and Faculty of Pharmaceutical Sciences, Sendai, Miyagi, Japan

6 Department of Nephrology and Rheumatology, Kagawa Prefectural Central Hospital, Takamatsu, Kagawa, Japan

7 Department of Nephrology, Hiroshima University Hospital, Hiroshima, Hiroshima, Japan
Nangaku, Hideki Hirakata, Takashi Wada, Terumasa Hayashi, Hiroshi Sato, Yasushi Yamazaki, Takao Masaki, Tatsuo Kagimura, Hiroyasu Yamamoto, Hiroki Hase, Masahiro Kamouchi, Enyu Imai, Kyoichi Mizuno, Manabu Iwasaki, Tadao Akizawa, Yoshiharu Tsubakihara, Shoichi Maruyama, and Ichiei Narita was originally published electronically on the publisher's internet portal (currently SpringerLink) on June 28, 2017 without open access.

With the author(s)' decision to opt for Open Choice the copyright of the article changed on August 15, 2017 to (C)

8 Translational Research Informatics Center, Foundation Biomedical Research and Innovation, Kobe, Hyogo, Japan

9 Department of Internal Medicine, Atsugi City Hospital, Atsugi, Kanagawa, Japan

10 Division of Nephrology, Toho University Ohashi Medical Center, Meguro, Tokyo, Japan

11 Department of Health Care Administration and Management, Graduate School of Medical Sciences, Kyushu University, Fukuoka, Fukuoka, Japan

12 Nakayamadera Imai Clinic, Takarazuka, Hyogo, Department of Nephrology, Fujita Health University Toyoake, Toyoake, Aichi, Japan

13 Mitsukoshi Health and Welfare Foundation, Shinjuku, Tokyo, Japan

14 Department of Computer and Information Science, Seikei University, Musashino, Tokyo, Japan

15 Division of Nephrology, Department of Medicine, Showa University School of Medicine, Shinagawa, Tokyo, Japan 
The Author(s) 2017 and the article is forthwith distributed under the terms of the Creative Commons Attribution 4.0 International License (http://creativecommons.org/licen ses/by/4.0/), which permits use, duplication, adaptation, distribution and reproduction in any medium or format, as long as you give appropriate credit to the original author(s) and the source, provide a link to the Creative Commons license and indicate if changes were made.

The original article was corrected.

16 Course of Safety Management in Health Care Sciences, Graduate School of Health Care Sciences, Jikei Institute, Yodogawa, Osaka, Japan

17 Department of Nephrology, Nagoya University Graduate School of Medicine, Nagoya, Aichi, Japan

18 Division of Clinical Nephrology and Rheumatology, Niigata University Graduate School of Medical and Dental Sciences, Niigata, Niigata, Japan 